\title{
A Pilot Study Comparing FLT-PET and FDG-PET in the Evaluation of Response to Cetuximab and Radiation Therapy in Advanced Head and Neck Malignancies
}

Brandon M. Barney ${ }^{1 *}$, Val Lowe ${ }^{2}$, Scott H. Okuno ${ }^{3}$, Bradley J. Kemp ${ }^{2}$, Mark S. Jacobson BA ${ }^{2}$, Katharine A. Price ${ }^{3}$, Jean E. Lewis ${ }^{4}$, Jan L. Kasperbauer ${ }^{5}$, Debra H. Brinkmann ${ }^{1}$, Robert L. Foote ${ }^{1}$, Yolanda I. Garces ${ }^{1}$, Wenting Wu ${ }^{6}$ and Jann N. Sarkaria ${ }^{1}$

${ }^{1}$ Department of Radiation Oncology, Mayo Clinic, Rochester, MN, USA

${ }^{2}$ Division of Nuclear Medicine, Mayo Clinic, Rochester, MN, USA

${ }^{3}$ Division of Medical Oncology, Mayo Clinic, Rochester, MN, USA

${ }^{4}$ Division of Anatomic Pathology, Mayo Clinic, Rochester, MN, USA

${ }^{5}$ Department of Otorhinolaryngology, Mayo Clinic, Rochester, MN, USA

${ }^{6}$ Division of Biomedical Statistics and Informatics, Mayo Clinic, Rochester, MN, USA

\begin{abstract}
Background: We prospectively compared FLT-PET and FDG-PET in evaluating response to cetuximab and chemoradiotherapy for HNSCC.

Methods: Six patients with HNSCC received cetuximab followed by chemoradiotherapy. Patients had FLTand FDG-PET scans at baseline, after cetuximab, and 2 weeks into chemoradiotherapy. Changes in SUV $\max _{\text {ax }}$ on successive scans were compared to baseline.

Results: After induction therapy, changes in SUV ranged from -2 to $32 \%$ for FLT-PET and -24 to $0 \%$ for FDG-

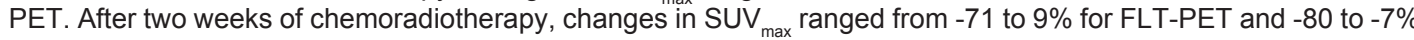
for FDG-PET. One patient experienced consecutive increases in FLT uptake not detected by FDG-PET. No patient recurred at a median 14.6 months.

Conclusions: Functional imaging early during definitive therapy for HNSCC is feasible. Similar changes in FLT and FDG uptake are detected during chemoradiotherapy; however, distinct differences were seen after induction cetuximab therapy. Further follow-up will facilitate correlation of radiotracer uptake with clinical outcome.
\end{abstract}

Keywords: Head and neck cancer; FDG-PET; FLT-PET; Cetuximab; Radiotherapy

\section{Introduction}

Nearly 40,000 people in the U.S. are diagnosed annually with locally advanced, potentially curable, head and neck squamous cell carcinoma [HNSCC], and most are treated with definitive chemoradiotherapy $[1,2]$. Despite improvements in systemic therapy and radiation targeting and delivery, rates of local failure approach $30 \%$ at 3 years $[3,4]$, and some patients require post-chemoradiotherapy neck dissection for persistent adenopathy or regional failure [5,6]. Thus, methods for early identification of tumors resistant to standard treatments would be helpful for individualizing therapies to maximize tumor control.

Both 2-deoxy-2-18 F-fluoro-D-glucose [FDG] positron emission tomography (PET) and 3'-deoxy-3'-18 F-fluorothymidine (FLT) PET imaging have been used in HNSCC to define disease extent and assess treatment response [7-11]. FLT-PET has a theoretical advantage over FDG-PET, since cellular uptake of FLT is a more specific cellular proliferation marker whereas FDG uptake provides a generalized assessment of metabolic state. A randomized clinical trial demonstrated a survival benefit for patients with HNSCC treated with radiotherapy and concurrent epidermal growth factor receptor (EGFR) inhibitor therapy compared to patients treated with radiotherapy alone [12], and pre-clinical studies have shown treatment with either radiotherapy or the anti-EGFR antibody cetuximab can result in a reduction in FLT accumulation within 48 hours $[13,14]$. In the current feasibility study, we evaluated the potential for FLT- and FDG-PET to detect an early response to induction cetuximab therapy and concurrent chemoradiotherapy.

\section{Materials and Methods}

\section{Patients}

This prospective study was approved by the Mayo Institutional Review Board, and written informed consent was obtained from all patients. Eligible patients had biopsy-proven HNSCC requiring treatment with chemoradiotherapy, and an ECOG performance status of 0 or 1 . Patients with a recent surgery or infection, a poorly controlled inflammatory process, or uncontrolled diabetes mellitus were excluded from the study. All patients underwent pre-treatment laboratory evaluation (complete blood count, creatinine, total bilirubin, and aspartate aminotransferase), baseline staging FDG-PET scan, and evaluation by radiation, medical, and surgical oncologists. No patient was excluded from analysis for ineligibility after giving informed consent.

*Corresponding author: Brandon Barney, Department of Radiation Oncology, Mayo Clinic, 200 First Street SW, Rochester, MN 55905, USA, Tel: 507-284-8227; Fax: 507-284-0079; E-mail: barney.brandon@mayo.edu

Received December 17, 2011; Accepted February 02, 2012; Published February 05, 2012

Citation: Barney BM, Lowe V, Okuno SH, Kemp BJ, Mark S. Jacobson BA, et al. (2012) A Pilot Study Comparing FLT-PET and FDG-PET in the Evaluation of Response to Cetuximab and Radiation Therapy in Advanced Head and Neck Malignancies. J Nucl Med Radiat Ther 3:120. doi:10.4172/2155-9619.1000120

Copyright: (c) 2012 Barney BM, et al. This is an open-access article distributed under the terms of the Creative Commons Attribution License, which permits unrestricted use, distribution, and reproduction in any medium, provided the original author and source are credited. 
Citation: Barney BM, Lowe V, Okuno SH, Kemp BJ, Mark S. Jacobson BA, et al. (2012) A Pilot Study Comparing FLT-PET and FDG-PET in the Evaluation of Response to Cetuximab and Radiation Therapy in Advanced Head and Neck Malignancies. J Nucl Med Radiat Ther 3:120. doi:10.4172/2155-9619.1000120

Page 2 of 6

\section{Study design}

All patients were treated with chemoradiotherapy with curative intent. Weekly intravenous cetuximab infusion was initiated two weeks prior to radiotherapy: loading dose of $400 \mathrm{mg} / \mathrm{m}^{2}$ and then weekly doses of $250 \mathrm{mg} / \mathrm{m}^{2}$ throughout radiation. Concurrent intensity-modulated radiation therapy was started with the third dose of cetuximab using an integrated boost of $70 \mathrm{~Gy}$ in 35 daily $2 \mathrm{~Gy}$ fractions to gross tumor plus margin and $63 \mathrm{~Gy}$ in 35 daily $1.8 \mathrm{~Gy}$ fractions to sub-clinical disease. Patients also received weekly intravenous cisplatin chemotherapy (30 $\mathrm{mg} / \mathrm{m}^{2} /$ weekly) starting concurrently with radiotherapy.

Baseline FLT- and FDG-PET scans were obtained after registration but prior to induction cetuximab. Staging FDG-PET scans obtained at Mayo Clinic within 14 days of registration were used when available. FLT- and FDG-PET scans were repeated after the second dose of cetuximab but before initiation of concurrent chemoradiotherapy, and again after two weeks of chemoradiotherapy, between fractions 10 and 15. An optional tumor biopsy was obtained after completion of induction cetuximab to obtain tissue for immunohistochemistry (IHC) analysis. IHC analysis was performed on the initial biopsy specimen only in patients who elected to undergo optional post-induction therapy biopsy as well. FDG-PET scans were performed six weeks and six months following completion of chemoradiotherapy. The entire treatment schema is summarized in Figure 1.

\section{PET synthesis and acquisition}

${ }^{18} \mathrm{~F}$ was produced at Mayo Clinic using a PETtrace Cyclotron (GE Medical Systems, Inc, Milwaukee, Wisconsin), and ${ }^{18} \mathrm{~F}$-labeled FDG was synthesized with the standard automated Hamacher method. All patients fasted for at least six hours before injection of FDG. Blood glucose was measured just prior FDG injection. Patients were rescheduled if blood glucose was $>200 \mathrm{mg} / \mathrm{dL}$.

${ }^{18} \mathrm{~F}-\mathrm{FLT}$ was synthesized as described using 5'-O-[4,4'dimethoxytriphenylmethyl]-2,3'-anhydrothymidine precursor [15]. The PET radiochemistry module is a fully-automated one-pot synthesizer using solid phase extraction for purification of the crude material. The product is formulated in sterile $0.9 \%$ sodium chloride and up to $10 \%$ ethanol and sterilized by filtration via a Millex $0.22 \mathrm{~mm}$ vented GS-filter. Radiochemical purity ( $>90 \%)$ is determined by high performance liquid chromatography (HPLC), and chemical purity by HPLC and TLC (thin layer chromatography).

PET image acquisition was performed on a GE RX or 690 PET/ CT scanner (GE Medical Systems). Dedicated head and neck PET images were obtained between 1 and 1.5 hours after intravenous injection of $740 \mathrm{MBq}$ of FDG or FLT, applying a 2-dimensional mode with a 5-minute acquisition per bed position. Emission images were reconstructed using iterative reconstruction, and emission data were corrected for scatter, random events, and dead-time losses using the manufacturer's software. Image pixel size of the dedicated head and neck images was $4.25 \mathrm{~mm}$, displayed in a 256x256 array with a $30 \mathrm{~cm}$ field of view. They were reconstructed using iterative reconstruction applying 3 iterations and 35 subsets. Full-width at half maximum of the Gaussian smoothing filter applied in reconstruction was $5 \mathrm{~mm}$.

For the integrated PET/CT scan, nonenhanced CT images were acquired with a helical mode with a detector-row configuration of $4 \times 5 \mathrm{~mm}$, a high-speed mode [pitch of $6: 1$ ], a gantry rotation of $0.8 \mathrm{~s}$, and a table speed of $30 \mathrm{~mm}$ per gantry rotation, $140 \mathrm{kVp}$, and $120 \mathrm{~mA}$ (180 mA for dedicated head and neck). The 5-mm-thick transaxial CT images were reconstructed at 4.25-mm intervals (transaxial) for fusion with the transaxial PET images.

\section{PET image analysis}

All FLT- and FDG-PET images were analyzed in transverse, coronal, and sagittal planes by a clinical investigator (BMB) and nuclear medicine specialist (VL) blinded to other clinical data. Qualitative image evaluation consisted of a slice-by-slice comparison of FLT- and FDG-PET scans for each time interval, noting similarities and differences in regions of increased radiotracer uptake. Quantitative evaluation was performed using the PET Edge Contour tool on a MIMvista workstation (MIM Software Inc., Cleveland, OH). A 3-dimensional region of interest (ROI) was generated using a gradientbased algorithm. A single ROI was placed around all areas of focally increased radiotracer uptake including the primary site and regional lymph nodes, and SUV maximum ( $\left.\mathrm{SUV}_{\max }\right)$, total lesion glycolysis (TLG; FDG-PET), total lesion proliferation (TLP; FLT-PET), and functional tumor volume (FTV) were calculated. Tumor response was scored using criterion proposed by the European Organization for Research and Treatment of Cancer (EORTC) for FDG and de Langen et al. for FLT, respectively [16,17]: partial metabolic response (PMR, $\geq 15 \%$ reduction in $\mathrm{SUV}_{\max }$ for FDG-PET, $\geq 20 \%$ reduction in $\mathrm{SUV}_{\max }$ for FLT-PET), stable metabolic disease (SMD, $\leq 25 \%$ increase to $<15 \%$ decrease in tumor SUV for FDG-PET or $\leq 25 \%$ increase to $<20 \%$

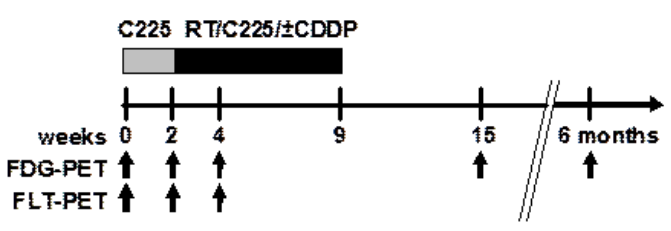

Figure 1: Treatment schema for this prospective study. FLT- and FDG-PET imaging was obtained at baseline, after 2 weeks of induction cetuximab (C225), and after 2 weeks of chemoradiotherapy (RT/C225/ \pm CDDP). FDG-PET scans were obtained 6 weeks and 6 months after completion of chemoradiation.

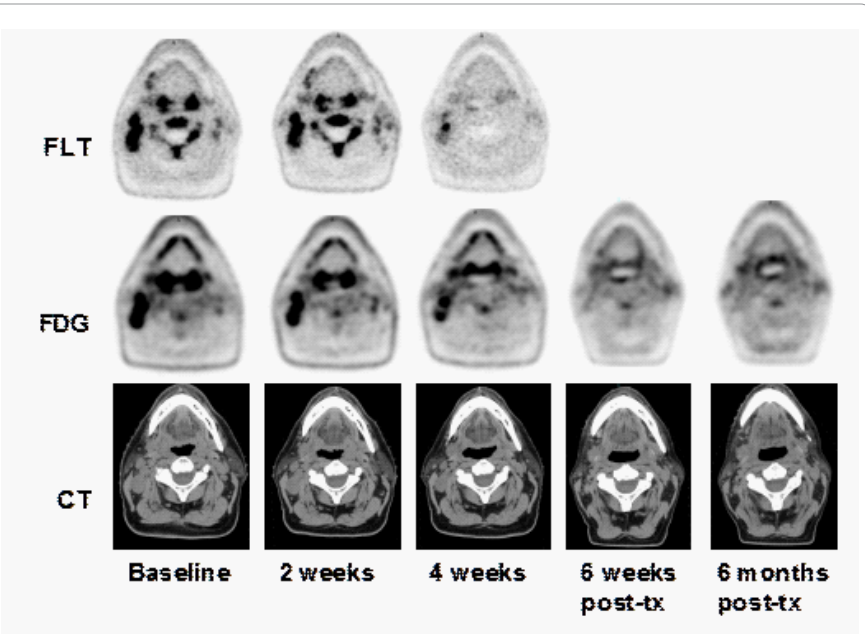

Figure 2: Representative FLT-PET, FDG-PET, and CT axial slices obtained through the primary tumor site and regional lymph nodes for Patient 1 . Both FLT- and FDG-PET showed SMD after induction therapy and PMR after 2 weeks of chemoradiotherapy. FDG-PET scans 6 weeks and 6 months after completion of chemoradiotherapy showed a continued decline in $\mathrm{SUV}_{\max }$. 
Citation: Barney BM, Lowe V, Okuno SH, Kemp BJ, Mark S. Jacobson BA, et al. (2012) A Pilot Study Comparing FLT-PET and FDG-PET in the Evaluation of Response to Cetuximab and Radiation Therapy in Advanced Head and Neck Malignancies. J Nucl Med Radiat Ther 3:120. doi:10.4172/2155-9619.1000120

Page 3 of 6

decrease in tumor SUV for FLT-PET), or progressive metabolic disease (PMD, >25\% increase in tumor SUV for FDG- or FLT-PET).

\section{Ki-67 Immunohistochemistry}

Tissue sections were deparaffinized and rehydrated in distilled water, and antigen retrieval was performed using preheated EDTA at 98C for 30 minutes. Using a Dako Autostainer (Dako, Carpenteria, $\mathrm{CA})$, tissue sections were treated with peroxidase block, rinsed, incubated with a 1:100 dilution of primary antibody MIB-1 clone (Dako) and subsequently with an Envision dual link secondary antibody (Dako). Sections were developed with the high-sensitivity diaminobenzidine $(\mathrm{DAB}+)$ chromogenic substrate system (Dako) for colorimetric visualization and counterstained with a modified Schmitt's hematoxylin. The proliferative index was graded in a semiquantitative fashion: low, $<5 \%$ of tumor nuclei staining; moderate, 5 to $20 \%$; and high, $>20 \%$.

\section{Statistical considerations}

The primary study endpoints were changes in FLT- and FDGSUV ${ }_{\max }$ compared to baseline on scans obtained after induction cetuximab and during chemoradiotherapy. Other endpoints included changes in TLG/TLP, FTV, Ki-67 reactivity after induction therapy, patterns of failure, and survival. All treatment outcomes were defined from the time of initial biopsy. All statistical analysis was performed with JMP 8.0 (SAS Institute Inc., Cary, NC, USA).

\section{Results}

Six patients were accrued between November 2008 and February 2010 and were available for evaluation. Baseline characteristics for these patients are summarized in Table 1. Protocol adherence was good for imaging at baseline and during radiotherapy, with all patients undergoing FLT- and FDG-PET scans at these intervals. Four of the six patients underwent FLT- and FDG-PET scanning after induction cetuximab therapy prior to starting radiation and three of these four patients also underwent a second tumor biopsy at that time. The other three patients refused repeat biopsy. Protocol adherence was good for post-treatment follow-up imaging: six patients had an FDG-PET scan six weeks post-radiotherapy, and five of six patients underwent FDG-PET imaging six months after radiotherapy. Figure 2 shows representative FLT-PET, FDG-PET and CT axial slices for Patient 1 from each imaging time point.

\section{Baseline imaging}

Assessment of disease extent with FLT-PET in all cases was concordant with the disease extent defined by FDG-PET both with respect to the primary site and regional adenopathy. The range in baseline FLT-SUV ${ }_{\max }$ was 5.8 to 14.8 (median, 10.9) and in baseline FDG-SUV was 12.4 to 34.7 (median, 17.5; Table 2). For each patient, baseline $\mathrm{SUV}_{\text {max }}$ was higher for FDG-PET than FLT-PET. Other values of interest for baseline images as well as images at other time intervals, including TLG, TLP, and FTV, are reported in Table 2. Thus, although FLT- and FDG-PET showed similar results in terms of disease staging, FDG uptake was greater than FLT uptake at baseline.

\section{Post-induction therapy imaging and IHC}

Four patients were evaluable for FLT and FDG imaging response after two weeks of cetuximab therapy. By the response criteria, three patients had SMD by FLT-PET scanning (change in FLT-SUV max $_{\text {of }}$ $-1 \%,-2 \%$, and $-2 \%)$, and one patient (Patient 4$)$ had PMD (32\% increase in FLT-SUV ${ }_{\max }$ ). One patient (Patient 2) had a PMR by FDG-PET scanning $\left(24 \%\right.$ decrease in FDG-SUV ${ }_{\max }$ ) and three patients had SMD $(-14 \%,-5 \%$, and $0 \%)$. A comparison of FLT- and FDG-SUV changes showed relatively poor association between the two imaging modalities (Figure 3). Three patients had post-cetuximab biopsies; in all three cases, Ki-67 reactivity remained moderate to high. Figure 4 shows an example of pre- and post- FLT-PET, FDG-PET, and Ki-67 IHC images for Patient 3. The other two patients who underwent post-cetuximab biopsies had similar IHC findings. In summary, metabolic response to cetuximab induction monotherapy was detected in one patient by

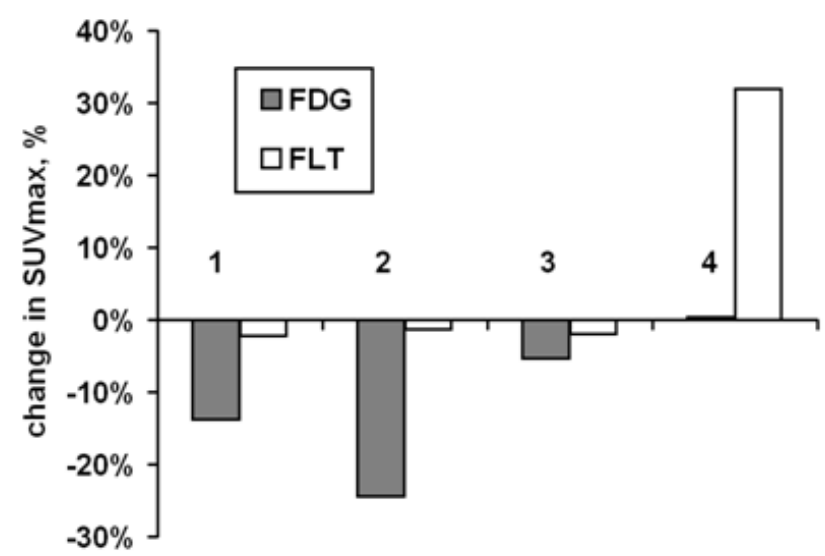

Figure 3: A modified waterfall plot of change in SUV after induction cetuximab for each patient. The white bars represent FLT-PET, and the grey bars represent FDG-PET. The numbers along the X-axis correspond to the patient number.

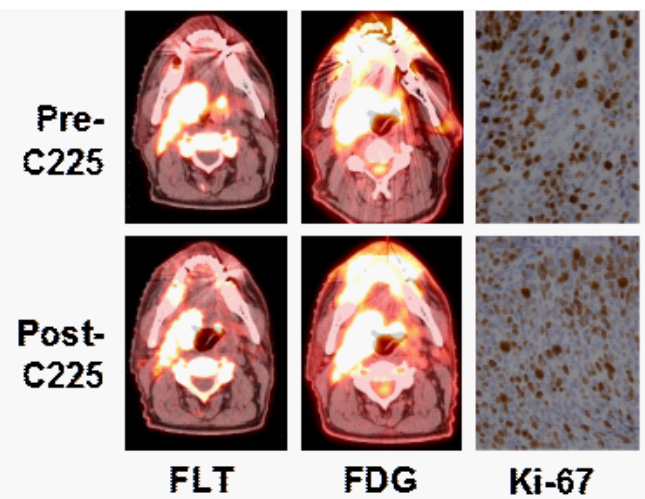

Figure 4: A comparison of fused FLT- and FDG-PET/CT scans and tumor Ki-67 immunohistochemistry for Patient 3 obtained before (pre-C225) and after (post-C225) induction cetuximab. FLT SUV max $_{1}$, FDG SUV max $_{\text {, }}$, and Ki67 proliferative index did not change significantly in response to induction therapy.

\begin{tabular}{|l|l|l|l|l|}
\hline Case & Age & Gender & Primary site & TNM stage \\
\hline 1 & 58 & M & Oropharynx & T4bN3M0 \\
\hline 2 & 77 & M & Oropharynx & T2N2bM0 \\
\hline 3 & 56 & M & Oropharynx & T3N2bM0 \\
\hline 4 & 42 & F & Oropharynx & T3N2bM0 \\
\hline 5 & 59 & M & Oropharynx & T4aN2bM0 \\
\hline 6 & 55 & M & Larynx & T3N0M0 \\
\hline
\end{tabular}

Table 1: Baseline characteristics for all six patients 
Citation: Barney BM, Lowe V, Okuno SH, Kemp BJ, Mark S. Jacobson BA, et al. (2012) A Pilot Study Comparing FLT-PET and FDG-PET in the Evaluation of Response to Cetuximab and Radiation Therapy in Advanced Head and Neck Malignancies. J Nucl Med Radiat Ther 3:120. doi:10.4172/2155-9619.1000120

Page 4 of 6

\begin{tabular}{|c|c|c|c|c|c|c|c|c|c|c|c|c|c|c|c|c|c|c|c|c|}
\hline \multirow[b]{3}{*}{ Case } & \multicolumn{6}{|c|}{ Baseline } & \multicolumn{6}{|c|}{ Post-cetuximab } & \multicolumn{6}{|c|}{ Intra-radiotherapy } & \multirow{2}{*}{\multicolumn{2}{|c|}{\begin{tabular}{|l} 
Post-treatment \\
FDG SUVmax
\end{tabular}}} \\
\hline & \multicolumn{2}{|c|}{ SUVmax } & \multicolumn{2}{|c|}{ TLG/TLP } & \multicolumn{2}{|c|}{ FTV } & \multicolumn{2}{|c|}{ SUVmax } & \multicolumn{2}{|c|}{ TLG/TLP } & \multicolumn{2}{|c|}{ FTV } & \multicolumn{2}{|c|}{ SUVmax } & \multicolumn{2}{|c|}{ TLG/TLP } & \multicolumn{2}{|c|}{ FTV } & & \\
\hline & FDG & FLT & FDG & FLT & FDG & FLT & FDG & FLT & FDG & FLT & FDG & FLT & FDG & FLT & FDG & FLT & FDG & FLT & 6 wks & $6 \mathrm{mos}$ \\
\hline 1 & 15.1 & 14.1 & 174.7 & 117.9 & 26.9 & 20.7 & 13.0 & 13.8 & 146.6 & 102.7 & 28.8 & 19.7 & 6.8 & 0.0 & 79.6 & 44.6 & 24.7 & 21.2 & 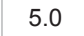 & 4.2 \\
\hline 2 & 20.9 & 14. & 97.9 & 43.4 & 11. & 10. & 15 & 1 & 5 & & 8.5 & & & 7.1 & 14 & 7.7 & 4.2 & 41 & 44 & N/A \\
\hline 3 & 13.4 & 7. & 22.4 & 113.9 & 23.4 & 33.5 & 12. & 6.9 & 113.7 & 108.6 & 23.3 & 34 & 2.9 & 3.3 & 21. & 6.8 & 17.7 & 20.1 & 5.8 & 6.0 \\
\hline 4 & 34.7 & 10.1 & 761.2 & 106.1 & 40.0 & 47.3 & 34.8 & 13.3 & 826.6 & 124.0 & 46.5 & 48.6 & 26.5 & 11.1 & 181.4 & 108.4 & 19.8 & 22.4 & 0.0 & 4.0 \\
\hline 5 & 12.4 & 5.8 & 195.2 & 101.1 & 33.5 & 36.4 & 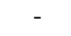 & 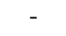 & - & 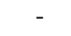 & - & - & 11.4 & 0.0 & 288.1 & 117.5 & 65.7 & 59.6 & 5.3 & 4.1 \\
\hline 6 & 19.8 & 11.7 & 125.3 & 65.9 & 13.6 & 13.8 & - & - & - & - & - & - & 3.9 & 3.4 & 45.8 & 14.7 & 24.9 & 11.8 & 4.4 & 4.5 \\
\hline
\end{tabular}

Table 2: SUV ${ }_{\max }$, TLG, TLP, and FTV for FDG-PET and FLT-PET scans at each time interval.

FDG-PET, while a different patient had metabolic progression detected by FLT-PET.

\section{Imaging during chemoradiotherapy}

All six patients were evaluable for FLT- and FDG-PET imaging response after two weeks of concurrent chemoradiotherapy. Compared to baseline, the changes in FLT-SUV ${ }_{\max }$ ranged from $-71 \%$ to $9 \%$ (median, -52.5\%), and the changes in FDG-SUV ${ }_{\text {max }}$ ranged from $-80 \%$ to $-7 \%$ (median, -55\%; Figure 5). A comparison of FLT- and FDG-PET showed generally good correlation between the two imaging studies, with the exception of Patient 4. In this patient, FLT SUV ${ }_{\max }$ increased by $9 \%$ compared to baseline, while the FDG SUV ${ }_{\max }$ changed by $-23 \%$. Although no clear cause for these deviations is evident, it is interesting to note that this patient was the only female studied and was by far the youngest patient on the study (42 years; Table 1 ). By response criteria, four of six patients had a PMR by FLT-PET compared to five of six with a PMR by FDG-PET. Only Patient 5 did not achieve a PMR by either FLT-PET (-9\% relative to baseline) or FDG-PET (-7\% relative to baseline). Therefore, after two weeks of induction cetuximab followed by two weeks of concurrent chemoradiotherapy, most patients had experienced a considerable decrease in tumor $\mathrm{SUV}_{\max }$ compared to baseline by both FLT- and FDG-PET.

\section{Post-treatment imaging and patient outcomes}

Patients were seen six weeks after completion of therapy and then at three month intervals for the first two years. No patient has been lost to follow-up at a median 26.1 months (range, 23.0 to 37.7 months). In all patients, FDG-PET imaging demonstrated a continued and marked SUV treatment response in both the primary site and regional lymph nodes at the six week post-treatment time interval, with $\mathrm{SUV}_{\max }$ ranging from 4.4 to 6.6 (median, 5.2). This response persisted at the six-month time interval in the five patients for whom six month FDGPET scans were available, with an $\mathrm{SUV}_{\text {max }}$ range of 4.0 to 6.0 (median, 4.2). These imaging findings were confirmed by history and physical examination at similar time intervals. The patient who did not have FDG-PET imaging at six months instead had a CT scan of the chest along with a comprehensive head and neck examination due to physician preference, as one of the patient's previous chest CT scans had shown indeterminate pulmonary nodules. Currently, this patient is clinically without evidence of disease, and the indeterminate lung findings continue to be stable. In all patients, no areas of new PET avidity, either in the head and neck or distant regions, have been noted on post-treatment imaging, no patient has required a neck dissection or other secondary surgical procedure.

\section{Discussion}

This pilot study demonstrates that similar changes in FLT and FDG uptake can be detected in HNSCC early during chemoradiotherapy. In the four patients examined after induction cetuximab, FLT uptake was unchanged in three patients and increased in one patient, while FDG uptake decreased in one of four patients. In contrast, after two weeks of concurrent chemoradiotherapy, both FLT and FDG uptake decreased in most patients, and the extent of change in FLT and FDG SUV ${ }_{\max }$ correlated closely in all but one patient. After completion of definitive therapy, a continued decline in FDG avidity was noted, with successive decrements in SUV ${ }_{\text {max }}$ noted at six week and six month time intervals. These data support the concept that functional imaging during treatment is feasible and may provide useful predictive information if these early findings ultimately correlate with patient outcome.

Agents targeting the EGFR signaling pathway can rapidly suppress metabolism and proliferation in responsive tumors, and our group and others have demonstrated suppression of FLT and FDG uptake within 48 hours of starting EGFR inhibitor therapy in xenograft models [14,18-21]. In clinical studies, a response to human epidermal growth factor receptor 2 (HER-2) inhibitor therapy has been monitored by FDG-PET, and data suggests a PMR four to six weeks after treatment initiation correlates with a prolonged progression-free interval with continued treatment $[22,23]$. Similarly, patients with lung cancer who underwent FLT-PET and had more than a $10.8 \%$ decrease in SUV 7 days after starting gefitinib (a small molecule EGFR inhibitor) were more likely to respond to therapy [24]. In the current study, a minimal change in FLT SUV ${ }_{\max }(-1$ to $-2 \%)$ was observed in three of the four patients following cetuximab induction therapy, while 1 patient had a $32 \%$ increase in $\mathrm{SUV}_{\max }$. Post-treatment biopsies in three of these patients demonstrated a moderate to high Ki-67 proliferative index, consistent with a lack of anti-proliferative activity of cetuximab. The elevated FLT uptake in the one patient falls outside the range of FLT variation previously described for serial scanning in NSCLC and may reflect a biological effect of cetuximab therapy [25]. It is also possible that the post-induction therapy imaging was performed prematurely and did not reflect the maximum benefit of cetuximab alone. Regardless of the mechanism underlying this singular response, both FLT-PET and Ki-67 analysis suggest that cetuximab did not significantly inhibit tumor cell proliferation in the four patients analyzed.

The current study is the first direct comparison of changes in FLT and FDG uptake in patients treated with a molecularly targeted therapeutic agent. FDG uptake is modulated by the level and activity of glucose transporters on the cell surface and cellular retention is ensured by phosphorylation of FDG by hexokinase. In contrast, FLT is transported into cells by nucleoside transporters and then trapped by thymidine kinase 1-mediated phosphorylation. Presumably, the discordant effects of cetuximab on FDG uptake versus FLT uptake in the current study reflects divergence of signaling pathways downstream of EGFR that impact on FDG versus FLT uptake and retention. This 
Citation: Barney BM, Lowe V, Okuno SH, Kemp BJ, Mark S. Jacobson BA, et al. (2012) A Pilot Study Comparing FLT-PET and FDG-PET in the Evaluation of Response to Cetuximab and Radiation Therapy in Advanced Head and Neck Malignancies. J Nucl Med Radiat Ther 3:120. doi:10.4172/2155-9619.1000120

Page 5 of 6



Figure 5: A modified waterfall plot of change in SUV at 4 weeks (after induction therapy and 2 weeks of chemoradiotherapy) for each patient. The white bars represent FLT-PET, and the grey bars represent FDG-PET. The numbers along the $\mathrm{X}$-axis correspond to the patient number.

finding supports the concept that different biological information may be gained from changes in both FLT and FDG uptake in the same patient after therapy with molecularly targeted agents.

FDG- and FLT-PET have both been used as early predictors of radiotherapy response, but this is the first study to directly compare these modalities in the same patient. Other groups have correlated FDG metabolic responses during radiotherapy with clinical outcomes for rectal, cervical, and non-small cell lung cancers [26]. Preliminary clinical studies of intra-radiotherapy FLT-PET in patients with esophageal, lung, and head and neck cancers have demonstrated consistent reduction in radiotracer uptake without attempting to correlate response with outcome $[7,10,27,28]$. Similarly, intraradiotherapy imaging in the current study was associated with a marked $\mathrm{SUV}_{\max }$ reduction within 10 to 15 fractions of radiation for both tracers. Concordance in radiotracer uptake between modalities was tighter at this interval for most patients, although one patient had stable FLT uptake with a contrasting reduction in FDG uptake. While the mechanism responsible for this finding is not known, 'flares' in radiotracer uptake have been observed in previous studies of intraradiotherapy FLT-PET imaging and may reflect accelerated tumor repopulation $[27,29]$. Additionally, one patient failed to achieve a PMR by either intra-radiotherapy PET modality. Based on the hypothesis that early PET imaging may facilitate identification of patients in whom radiation will be ineffective, the clinical outcomes for these 2 patients lacking a PMR by FLT and/or FDG during radiotherapy will be quite interesting. Currently, neither patient has manifested evidence of tumor recurrence.

Potential study weaknesses include small patient numbers and a lack of long-term follow-up, both of which prohibit a definitive statement regarding the capability of early functional imaging to predict for longterm clinical outcomes. Additionally, another potential limitation is the inherent variability observed with both FLT- and FDG-PET. Recent reports show that tumor volume and SUV may vary by as much as $30 \%$ on a day-to-day basis with both FLT- and FDG-PET, even without treatment $[30,31]$. Yet even if all differences of $<30 \%$ in $\mathrm{SUV}_{\max }$ were assumed to represent random day-to-day SUV variation rather than measurable treatment responses, the SUV changes measured by FLT- and FDG-PET in Patient 4 would be dissimilar enough to warrant discussion. By the same token, using this criteria, changes in SUV for all other patient scans would fall within the $+/-30 \%$ threshold, implying FLT and FDG provided similar information for these patient's tumors.

\section{References}

1. Jemal A, Siegel R, Xu J, Ward E (2010) Cancer statistics. CA Cancer J Clin 60: $277-300$

2. Altekruse SF, Kosary CL, Krapcho M, Neyman N, Aminou R, et al. (2010) SEER Cancer Statistics Review, 1975-2007. National Cancer Institute.

3. Ang K, Pajak T, Wheeler R, D. Rosenthal, F. Nguyen-Tan, et al. (2010) A phase III trial to test accelerated versus standard fractionation in combination with concurrent cisplatin for head and neck carcinomas [RTOG 0129]: report of efficacy and toxicity. International Journal of Radiation Oncology, biology, physics $77: 1-2$.

4. Forastiere AA, Goepfert H, Maor M, Pajak TF, Weber R, et al. (2003) Concurrent chemotherapy and radiotherapy for organ preservation in advanced laryngeal cancer. N Engl J Med 349: 2091-2098.

5. Forest VI, Nguyen-Tan PF, Tabet JC, Olivier MJ, Larochelle D, et al. (2006) Role of neck dissection following concurrent chemoradiation for advanced head and neck carcinoma. Head Neck 28: 1099-1105.

6. Corry J, Peters L, Fisher R, Macann A, Jackson M, et al. (2008) N2-N3 neck nodal control without planned neck dissection for clinical/radiologic complete responders-results of Trans Tasman Radiation Oncology Group Study 98.02. Head Neck 30: 737-742.

7. Menda Y, Boles Ponto LL, Dornfeld KJ, Tewson TJ, Watkins GL, et al. (2009) Kinetic analysis of 3'-deoxy-3'-[18]F-fluorothymidine [[18]F-FLT] in head and neck cancer patients before and early after initiation of chemoradiation therapy. J Nucl Med 50: 1028-1035.

8. Nam SY, Lee SW, Im KC, Kim JS, Kim SY, et al. (2005) Early evaluation of the response to radiotherapy of patients with squamous cell carcinoma of the head and neck using 18FDG-PET. Oral oncol 41: 390-395.

9. Rege S, Maass A, Chaiken L, Tewson TJ, Watkins GL, et al. (1994) Use of positron emission tomography with fluorodeoxyglucose in patients with extracranial head and neck cancers. Cancer 73: 3047-3058.

10. Troost EG, Bussink J, Hoffmann AL, Boerman OC, Oyen WJ, et al. (2010) $18 \mathrm{~F}-\mathrm{FLT} \mathrm{PET} / \mathrm{CT}$ for early response monitoring and dose escalation in oropharyngeal tumors. J Nucl Med 51: 866-874.

11. Yao M, Graham MM, Smith RB, Dornfeld KJ, Skwarchuk M, et al. (2004) Value of FDG PET in assessment of treatment response and surveillance in head-and-neck cancer patients after intensity modulated radiation treatment: a preliminary report. Int J Radiat Oncol Biol Phys 60: 1410-1418.

12. Bonner JA, Harari PM, Giralt J, Azarnia N, Shin DM, et al. (2006) Radiotherapy plus cetuximab for squamous-cell carcinoma of the head and neck. $\mathrm{N}$ Engl $J$ Med 354: 567-578.

13. Sugiyama M, Sakahara H, Sato K, Harada N, Fukumoto D, et al. (2004) Evaluation of 3'-deoxy-3'-18F-fluorothymidine for monitoring tumor response to radiotherapy and photodynamic therapy in mice. J Nucl Med 45: 1754-1758.

14. Waldherr C, Mellinghoff IK, Tran C, Halpern BS, Rozengurt N, et al. (2005) Monitoring antiproliferative responses to kinase inhibitor therapy in mice with 3'-deoxy-3'-18F-fluorothymidine PET. J Nucl Med 46: 114-120.

15. Machulla HJ, Blocher A, Kuntzsch M ( 2000) Simplified labeling approach for synthesizing 3 ,-deoxy-3,-[F-18]fluorothymidine [[F-18]FLT]. 243: 843-846.

16. Young H, Baum R, Cremerius U, Herholz K, Hoekstra O, et al. (1999) Measurement of clinical and subclinical tumour response using [18F]fluorodeoxyglucose and positron emission tomography: review and 1999 EORTC recommendations. European Organization for Research and Treatment of Cancer (EORTC) PET Study Group. Eur J Cancer 35: 1773-1782.

17. de Langen AJ, Klabbers B, Lubberink M, Boellaard R, Spreeuwenberg MD et al. (2009) Reproducibility of quantitative 18F-3'-deoxy-3'-fluorothymidine measurements using positron emission tomography. Eur $\mathrm{J}$ Nucl Med Mol Imaging 36: 389-395

18. Atkinson DM, Clarke MJ, Mladek AC, Carlson BL, Trump DP, et al. (2008) Using fluorodeoxythymidine to monitor anti-EGFR inhibitor therapy in squamous cel carcinoma xenografts. Head Neck 30: 790-799.

19. Ullrich RT, Zander T, Neumaier B, Koker M, Shimamura T, et al. (2008) Early detection of erlotinib treatment response in NSCLC by 3'-deoxy-3'-[F]-fluoroL-thymidine [[F]FLT] positron emission tomography [PET]. PloS one 3: e3908.

20. Su H, Bodenstein C, Dumont RA, Seimbille Y, Dubinett S, et al. (2006) 
Citation: Barney BM, Lowe V, Okuno SH, Kemp BJ, Mark S. Jacobson BA, et al. (2012) A Pilot Study Comparing FLT-PET and FDG-PET in the Evaluation of Response to Cetuximab and Radiation Therapy in Advanced Head and Neck Malignancies. J Nucl Med Radiat Ther 3:120. doi:10.4172/2155-9619.1000120

Page 6 of 6

Monitoring tumor glucose utilization by positron emission tomography for the prediction of treatment response to epidermal growth factor receptor kinase inhibitors. Clin Cancer Res 12: 5659-5667.

21. Dorow DS, Cullinane C, Conus N, Roselt P, Binns D, et al. (2006) Multi-trace small animal PET imaging of the tumour response to the novel pan-Erb-B inhibitor Cl-1033. Eur J Nucl Med Mol Imaging 33: 441-452.

22. Herbst RS, Davies AM, Natale RB, Dang TP, Schiller JH, et al. (2007) Efficacy and safety of single-agent pertuzumab, a human epidermal receptor dimerization inhibitor, in patients with non small cell lung cancer. Clin Cancer Res 13: 6175-6181.

23. Kawada K, Murakami K, Sato T, Kojima Y, Ebi H, et al. (2007) Prospective study of positron emission tomography for evaluation of the activity of lapatinib a dual inhibitor of the ErbB1 and ErbB2 tyrosine kinases, in patients with advanced tumors. Jpn lin Oncol 37: 44-48.

24. Sohn HJ, Yang YJ, Ryu JS, Oh SJ, Im KC, et al. (2008) [18F]Fluorothymidine positron emission tomography before and 7 days after gefitinib treatment predicts response in patients with advanced adenocarcinoma of the lung. Clin Cancer Res 14: 7423-7429.

25. Shields AF, Lawhorn-Crews JM, Briston DA, Zalzala S, Gadgeel S, et al. (2008) Analysis and reproducibility of 3'-Deoxy-3'-[18F]fluorothymidine positron emission tomography imaging in patients with non-small cell lung cancer. Clin Cancer Res 14: 4463-4468.

26. Kong FM, Frey KA, Quint LE, Ten Haken RK, Hayman JA, et al. (2007) A pilot study of [18F]fluorodeoxyglucose positron emission tomography scans during and after radiation-based therapy in patients with non small-cell lung cancer. $J$ Clin Oncol 25: 3116-3123.

27. Yue J, Chen L, Cabrera AR, Sun X, Zhao S, et al. (2010) Measuring tumor cell proliferation with 18F-FLT PET during radiotherapy of esophageal squamous cell carcinoma: a pilot clinical study. J Nucl Med 51: 528-534.

28. Everitt S, Hicks RJ, Ball D, Kron T, Schneider-Kolsky M, et al. (2009) Imaging cellular proliferation during chemo-radiotherapy: a pilot study of serial $18 \mathrm{~F}-\mathrm{FLT}$ positron emission tomography/computed tomography imaging for non-smallcell lung cancer. Int J Radiat Oncol Biol Phys 75: 1098-1104.

29. Withers HR, Taylor JM (1993) Critical volume model.Int J Radiat Oncol Bio Phys 25: 151-152.

30. Frings $V$, de Langen AJ, Smit EF, van Velden FH, Hoekstra OS, et al. (2010) Repeatability of metabolically active volume measurements with 18F-FDG and 18F-FLT PET in non-small cell lung cancer. J Nucl Med 51: 1870-1877.

31. Hatt M, Cheze-Le Rest C, Aboagye EO, Kenny LM, Rosso L, et al. (2010) Reproducibility of 18F-FDG and 3'-deoxy-3'-18F-fluorothymidine PET tumor volume measurements. J Nucl Med 51: 1368-1376. 